\title{
Systematic Review and Meta-analysis of Real-time Polymerase Chain Reaction assay for the detection of COVID-19 from clinical samples in low-and middle-income countries: Protocol
}

Emmanuel Oladipo Babafemi ( $\sim$ boladiipo95@yahoo.com )

\section{Research}

Keywords: COVID-19, Systematic review, Meta-analysis, Real-time Polymerase Chain Reaction, Low-and middle-income countries

Posted Date: October 8th, 2021

DOl: https://doi.org/10.21203/rs.3.rs-960246/v1

License: (c) (i) This work is licensed under a Creative Commons Attribution 4.0 International License. Read Full License 
1 COVID-19 in LMICs PROTOCOL

2 Title:

3 Systematic Review and Meta-analysis of Real-time Polymerase Chain Reaction

4 assay for the detection of COVID-19 from clinical samples in low-and middle-income

5 countries: Protocol

6 Emmanuel O Babafemi ${ }^{1}$, Benny P Cherian², Gilbert Mogoko ${ }^{3}$

7 Author details:

8 1Emmanuel O. Babafemi: E.O.Babafemi@limu.ac.uk- Corresponding author

Pharmacy and Biomolecular Sciences Room 10.01, James Parsons Building Byrom Street, Liverpool John Moores University, Liverpool, L3 3AF, United Kingdom

²Benny P. Cherian: benny.cherian@nhs.net

Infection Control Doctor/Consultant Microbiologist, Barts Health NHS Trust Room MICR324,3rd floor Pathology \& Pharmacy Building, 80 Newark Street, London E1 2ES, United Kingdom

${ }^{3}$ Gilbert Mogoko: G_mogoko@yahoo.co.uk Microbiology Department IPP Pathology First, Dobson House, Bentalls, Basildon SS14 3BY, United Kingdom

\section{Abstract}

Background:

COVID-19 has spread globally since its discovery in Hubei province, China in December 2019 and became pandemic in 2020. COVID-19 is a new betacoronavirus and a variant of severe acute respiratory syndrome coronavirus 2 (SARA- CoV-2). Rapid, accurate and reliable diagnosis of COVID-19 will prevent the spread and allow for appropriate management. The main objective of this systematic review is to identify, appraise and summarise the published evidence on the diagnostic performance and effectiveness of SARS-CoV-2 virus in the diagnosis of current or previous COVID-19 using real-time polymerase chain reaction (RT-PCR) assay in low-and middle-income countries (LMICs).

Methods: We will search MEDLINE/PubMed, EMBASE, BIOSIS, LILACS, Cochrane Infectious Diseases Group Specialised Register (CIDG SR), Global Health, and CINAHL for published studies for the diagnosis of COVID-19 using realtime polymerase chain reaction assay in LMICs

There will be no restriction regarding the language, date of publication, and publication status. We will include retrospective, cross-sectional and cohort observational studies will be included in the review.

Selection of studies, data extraction and management, assessment of risk of bias, and quality of evidence will be performed by two independent reviewers (EB and $\mathrm{BC})$. A third researcher (GM) will be consulted in case of discrepancies. Depending on the availability and quality of the data, a meta-analysis will be performed.

Otherwise, findings will be qualitatively reported. 
Discussion: To our knowledge, this is the first systematic review and meta-analysis to assess the uptake of RT-PCR assay for SARS-CoV-2 detection from clinical samples in human in LMICs. This review will make available evidence on the uptake, accuracy, approach, and interpretation of results of this assay in the context of COVID-19 diagnosis which will meet an urgent need, considering the diagnostic challenges of RT-PCR assay for COVID-19 diagnosis in humans.

Systematic review registration: PROSPERO CRD42021271894

Keywords: COVID-19; Systematic review; Meta-analysis; Real-time Polymerase Chain Reaction; Low-and middle-income countries

\section{Background}

Since the first discovery and reported case of a novel coronavirus in Wuhan, China in December 2019, which spread rapidly across the world. The virus was named severe acute respiratory syndrome coronavirus 2 (SARS-CoV-2), and the disease that it causes, COVID-19. Since then, several countries have seen a rise of confirmed cases, first coming from cross-border travel and later by subsequent community transmission [1]. In the early stage of the pandemic, the World Health Organization (WHO) stated that testing for the virus should be considered for symptomatic patients on the basis of the suspicion and likelihood of COVID-19, as well as in those who are asymptomatic or minimally symptomatic but who have been in contact with confirmed cases [2]. SARS-CoV-2 testing is a major bottleneck globally, especially in low- and middle-income countries (LMICs). As the public health sector struggles to meet the increasing need for tests, LMICs are now seeing a rise in COVID-19 cases, particularly in South Asia, South America, and Africa [3] Globally, $70 \%$ of decisions with patient care are based on laboratory results [4]. Accurate diagnosis of COVID-19 is a key component in addressing the pandemic. Diagnostics can play an important role in the containment of COVID-19, enabling the rapid implementation of control measures that limit the spread through case identification, isolation, and contact tracing (i.e., identifying people that may have come in contact with an infected patient).

Diagnosis of COVID-19 in LMICs is confronted with major challenges such as limited resources, inadequate capacity, untrained laboratory personnel, lack of laboratory personnel in testing facilities despite having a pool of unemployed and qualified experts, inadequate funding and lack of policies. Other challenges include the limited number of laboratories with the appropriate biosafety level (BSL) classification and available safety cabinets for processing samples associated with SARS-CoV-2, obtaining governmental approval to conduct SARS-CoV-2 testing remains challenging, the high cost of testing and the high patient-borne costs present a major barrier to testing in LMICs.

SARS-CoV-2 has a single-stranded positive sense RNA genome that is $\sim 30,000$ nucleotides in length $[5,6]$. Of 104 strains sequenced between December 2019 and mid-February 2020, 99.9\% sequence homology was observed, but, more recently, 
changes in the viral genome have been catalogued, showing a higher sequence diversity $[7,8]$.

Nucleic acid testing is the primary method of diagnosing COVID-19 [9]. A number of reverse transcription polymerase chain reaction kits have been designed to detect SARS-CoV-2 genetically. RT-PCR involves the reverse transcription of SARS-CoV-2 RNA into complementary DNA (cDNA) strands, followed by amplification of specific regions of the CDNA $[10,11]$.

Reverse-transcriptase polymerase chain reaction assay detects the presence of SARS-CoV-2 virus usually through the use of methods that recognise and amplify SARS-CoV-2 viral nucleic acid. SARS CoV-2 virus testing is usually done in a specialised laboratory setting using respiratory samples, such as nasopharyngeal swabs.

The purpose of this systematic review is to identify, appraise and summarise the published evidence on the diagnostic performance and effectiveness of SARS-CoV2 virus in the diagnosis of current or previous COVID-19 using real-time polymerase chain reaction (RT-PCR) platform which is the current gold standard for diagnosis. The review will also explore the uptakes of this RT-PCR assay in LMICs for the testing/diagnosis across different continents of the globe, and whether testing is laboratory based or done at point of care.

\section{Research question}

How accessible is Real-time Polymerase Chain Reaction assays for the diagnosis of COVID-19 in all clinical samples in low-and middle-income countries?

\section{Methods}

This systematic review protocol has been developed based on the Preferred Reporting Items for Systematic Reviews and Meta-Analyses Protocols (PRISMA-P) guidelines [18], which is available in Additional file 1. The systematic review protocol was registered with the International Prospective Register of Systematic Reviews (PROSPERO) database (registration ID: CRD42021271894). We will search MEDLINE/PubMed, EMBASE, BIOSIS, LILACS, Cochrane Infectious Diseases Group Specialised Register (CIDG SR), Global Health, and CINAHL using the search strategy and terms used for one of the databases as detailed in Additional file 2. This will be used for published studies that used RT-PCR assays for detecting SARS-CoV-2 in LMICs. The electronic search will be tailored for each database to include its specific keywords and MeSH terms.

\section{Searching other resources}

To avoid missing relevant studies to be included, searching other sources by looking through reference lists of relevant reviews and selected studies, searching websites of a relevant organization, performing forward citation searching of relevant articles using the PubMed related articles feature, Google Scholar, Cochrane Library, turning research into practice (TRIP), dissertations, Conference Proceedings Citation Index - Science (CPCI-S), the portal of the WHO International Clinical Trials Registry Platform (www.who.int/trialsearch) to identify ongoing trials, the World Health Organization and Centers for Disease Control and Prevention websites. We will also contact leading researchers at the Foundation for Innovative New Diagnostics (FIND). There will be no restriction regarding the language, date of 
publication and publication status. A search of grey literature and theses databases

\section{Data collection and analysis}

\section{Study selection and data extraction}

The two review authors (EB and BC) will independently screen for eligible studies after the literature search. Following screening, selection of studies irrespective of their design provided they meet the inclusion criteria will be carried out by two authors (EB and BC). They will independently review titles and abstracts against eligibility criteria to categorise as either 'potentially include' or 'exclude' (see Additional file 3, which is the flow chart diagram). A third researcher (GM) will be consulted in case of discrepancies at each of the stages. We will resolve differences in opinion through discussion. We will list studies excluded after full-text assessment and their reasons for exclusion in a 'Characteristics of excluded studies' table. Data will be extracted independently by EB \& BC for qualitative and quantitative parameters from each selected study using a predetermined list of categories/characteristics: first author, year of publication participants/population, index test, limit of detection, country of origin of the study, type of study, type of sample, disease and target sequence gene for COVID-19 RNA detection and results into a standardised data extraction form (see Additional file 4 Part $A$ ). We will conduct a risk of bias assessment at the level of the study using QUADAS-2 (University of Bristol) tool that assesses diagnostic evaluation work in four domains: (1) patient selection, (2) the index test, (3) the reference standard, and (4) patient flow and timing of tests (see Additional file 4 Part B). Discordant results will be resolved by consensus or through a third investigator-GM. The findings of the systematic search will be presented in the prospective systematic review in a flow diagram according to the 'Preferred Reporting Items for Systematic Reviews and Meta-analyses' (PRISMA) 2020 statement.

We will utilise the Review Manager (RevMan V5.4, Cochrane Collaboration, Oxford, UK) and Meta-DiSC (version 2.0) statistical software to carry out the meta-analysis $[12,13]$. We will also report point estimates and 95\% confidence intervals, for sensitivity and specificity for each study and for pooled data, using bivariate randomeffects meta-analysis. We will report these results using a forest plot and plot a summary receiver operating characteristics (SROC) curve [14, 15]. Heterogeneity between the studies in effect measures will be assessed using both the $Q$-test statistic and the $I$-Squared $\left(I^{2}\right)$ statistic. We will consider a Q-test with a p-value $<0.05$ and $R$ statistic of $>50 \%$ as indicative of substantial heterogeneity.

\section{Subgroup analyses}

Subgroup analyses or subsets potential heterogeneity will be performed if there is enough information/data using the following a priori that would enable us to categorise the whole tested population into subgroups such age, we will assess the performance of different types of RT-PCR assays used for the detection of SARSCoV-2 'RNA' or 'proteins'/COVID-19 from all the clinical specimen types and their respective target sequence genes, we will assess the uptake of RT-PCR assays in LMICs across various continents. We will assess sources of data to these graders.

\section{Quality assessment}


Two review authors (EB and $\mathrm{BC}$ ) will independently conduct a risk of bias assessment at the level of the study using the QUADAS-2 (University of Bristol), the recommended tool for evaluating primary studies for the inclusion in systematic reviews for diagnostic accuracy. QUADAS-2 tool with assessment based on risk of bias and applicability of results has four domains evaluating (1) patient selection, (2) the index test, (3) the reference standard, and (4) patient flow and timing of tests (see Additional file Part B)

\section{Assessment for heterogeneity and publication bias}

We will assess the extent of heterogeneity among studies visually with forest plots and SROC curves with 95\% prediction regions and statistically with chi-squared $\left(X^{2}\right)$ and I-squared $\left(I^{2}\right)[14,15]$. The source of heterogeneity will be investigated using stratified (subgroup) analyses. Every effort will be made to identify unpublished studies through searching conference abstracts, grey literature, and reference lists of relevant primary articles to minimise publication bias. Formal assessment of publication bias using methods such as funnel plots or regression tests was not evaluated as this is not usually recommended in the meta-analysis for diagnostic test accuracy $[14,15]$.

\section{Discussion}

To our knowledge, this is the first systematic review and meta-analysis to assess the uptake of RT-PCR assay for SARS-CoV-2 detection from clinical samples in human in LMICs. Pooling all available evidence on the accuracy, approach, and interpretation of results of this assay in the context of COVID-19 diagnosis will meet an urgent need, considering the challenges of COVID-19 diagnosis in LMICs. This we believe will strengthen the control of COVID-19 pandemic in LMICs. We therefore believe that our findings will have impact on policy and guide governmental \& nongovernmental organisations and other stakeholders to support clinical laboratory practice to provide affordable, improved and accurate COVID-19 diagnostic approach/protocol in LMICs. The practicality of using RT-PCR assays in a resource limited settings will be discussed within the technical challenges, cost, reagents, and other logistics. Strengths and limitations of included studies and this review will be discussed, and recommendations for further research and clinical practice will be provided.

\section{Additional files}

Additional file 1: PRISMA-P 2015 Checklist. (DOCX $33 \mathrm{~kb}$ )

Additional file 2: Search Strategy. (DOCX $14 \mathrm{~kb}$ )

Additional file 3: Flow Chart diagram. (DOC $60 \mathrm{~kb}$ )

Additional file 4: Part A: Data Extraction form file 4. Part B: QUADAS-2 (Quality assessment of diagnostic accuracy studies-2 tool). (DOCX $24 \mathrm{~kb}$ )

\section{Abbreviations}


cDNA: complementary deoxyribonucleic acid; CPCI-S: Conference Proceedings Citation Index-Science; COVID-19: Corona virus disease or 2019 novel coronavirus or 2019-nCoV; EMBASE: Excerpta Medica database; FIND : Foundation for Innovative New Diagnostics; GRADE: Grades of Recommendation, Assessment, Development and Evaluation; LMICs: low-and middle-income countries; LTBI: Latent tuberculosis infection; MEDLINE: Medical Literature Analysis and Retrieval System Online; MeSH: Medical Subject Headings; PRISMA: Preferred Reporting Items for Systematic Reviews and Meta-Analysis; PRISMA -P: Preferred Reporting Items for Systematic Reviews and Meta-Analysis Protocols; QUADAS-2: Quality assessment of diagnostic accuracy studies-2 tool; RNA: Ribonucleic acid; SARS CoV-2: Severe acute respiratory syndrome coronavirus 2; SROC: summary receiver operating characteristics; TRIP: Turning research into practice; WHO ICTRP: WHO International Clinical Trials Registry Platform.

\section{Acknowledgements} None

\section{Authors' contributions}

EB designed the systematic review protocol. EB and $\mathrm{BC}$ designed the search strategy for this systematic review protocol and performed the search. EB, BC and GM will be responsible for the data selection, data extraction, data analysis, and interpretation of the results. All authors critically revised the current protocol. All authors read and approved the final manuscript.

Funding

None

Ethics approval and consent to participate Not applicable Consent for publication

All authors have given consent and approval for the manuscript to be submitted for publication.

\section{Competing interests}

The authors declare that they have no competing interests.

Author details 1 School of Pharmacy and Biomolecular Sciences, Liverpool John Moores University, Byrom Street, L3 3FA, Liverpool, UK. 2 Barts Health NHS Trust, Room MICR324, 3rd floor Pathology \& Pharmacy Building, 80 Newark Street, London E1 2ES, UK. 3 Microbiology Department, IPP Pathology First, Dobson House, Bentalls, Basildon SS14 3BY, UK.

\section{References:}

258 1. Patel P, Adebisi YA, Steven M, et al. Addressing COVID-19 in Malawi. Pan Afr 
2. World Health Organization. Laboratory testing strategy recommendations for

COVID-19: interim guidance, 2020. Available: https://apps.who.int/iris/ handle/10665/331509 [Accessed 24 August 2021] 3. Nkengasong JN, Mankoula W. Looming threat of COVID-19 infection in Africa: act collectively, and fast. Lancet. Published online February 27, 2020. doi:10.1016/S0140-6736(20)30464-5

4. Lippi G, Plebani M. The add value of laboratory diagnostics: the many reasons why decision-makers should actually care. J Lab Precis Med 2017; 2:100

5. Zhou P, Yang XL, Wang XG, Hu B, Zhang L, Zhang W, Si HR, Zhu Y, Li B, Huang $\mathrm{CL}$, et al. A Pneumonia Outbreak Associated with a New Coronavirus of Probable Bat Origin. Nature 2020; 579: 270.10.1038/s41586-020-2012-7.

6. Wu A, Peng Y, Huang B, Ding X, Wang X, Niu P, Meng J, Zhu Z, Zhang Z, Wang $J$. Commentary Genome Composition and Divergence of the Novel Coronavirus (2019-nCoV) Originating in China. Cell Host Microbe 2020; 27: 325.10.1016/j.chom.2020.02.001.

7. Tang X, Wu C, Li X, Song Y, Yao X, Wu X, Duan Y, Zhang H, Wang Y, Qian Z, et al. On the Origin and Continuing Evolution of SARS-CoV-2. National Science Review 2020; 10.1093/nsr/nwaa036.

8. Report of the WHO-China Joint Mission on Coronavirus Disease 2019 (COVID19); WHO: Geneva, Switzerland, 2020.

9. CDC 2019-Novel Coronavirus (2019-nCoV) Real-Time RT-PCR Diagnostic Panel; Division of Viral Diseases, U.S. Centers for Disease Control and Prevention: Atlanta, GA, 2020.

10. Freeman WM, Walker SJ, Vrana KE. Quantitative RT-PCR: Pitfalls and Potential. BioTechniques 1999; 26(1): 124-125. 10.2144/99261rv01.

11. Kageyama T, Kojima S, Shinohara M, Uchida K, Fukushi S, Hoshino FB, Takeda N, Katayama K. Broadly Reactive and Highly Sensitive Assay for Norwalk-like Viruses Based on Real-Time Quantitative Reverse Transcription-PCR. J. Clin. Microbiol. 2003; 41(4): 1548-1557. 10.1128/JCM.41.4.1548-1557.2003. 12. DerSimonian R, Laird N. Meta-analysis in clinical trials. Control Clin. Trials. 1986;7(3):177-88.

13. Zamora J, et al. Meta-DiSc: a software for meta-analysis of test accuracy data. BMC Med Res Methodol. 2006; 6:31-42.

14. Leeflang MM, Deeks JJ, Takwoingi Y, Macaskill P. Cochrane diagnostic test accuracy reviews. Systematic reviews. 2013; 2:82. https://doi.org/10.1186/2 0464053-2-82.

15. Higgins JP, Green S. Cochrane handbook for systematic reviews of interventions. 2011. Retrieved July 3, 2015, from http://handbook.cochrane. org/handbook 


\section{Supplementary Files}

This is a list of supplementary files associated with this preprint. Click to download.

- Additionalfile1.pdf

- Additionalfile2.pdf

- Additionalfile3.pdf

- Additionalfile4PartAandB.pdf 